\title{
Probabilistic reversing operation with fidelity and purity gain for macroscopic quantum superposition
}

\author{
Hiroaki Terashima ${ }^{1,2}$ and Masahito Ueda ${ }^{1,2}$ \\ ${ }^{1}$ Department of Physics, Tokyo Institute of Technology, \\ Tokyo 152-8551, Japan \\ ${ }^{2}$ CREST, Japan Science and Technology Corporation (JST), \\ Saitama 332-0012, Japan
}

\begin{abstract}
It is shown that a large class of weak disturbances on macroscopic quantum superpositions can be canceled by a probabilistic reversing operation on the system. We illustrate this for spin systems undergoing an Ising-type interaction with the environment and demonstrate that both the fidelity to the original state and the purity of the amended state can simultaneously be increased by the reversing operation. A possible experimental scheme to implement our scheme is discussed.
\end{abstract}

PACS: 03.67.-a, 03.67.Pp, 03.65.Ta

Keywords: quantum information, quantum measurement, macroscopic quantum system

\section{Introduction}

The macroscopic quantum superpositions referred to as Schrödinger cat states are of great interest in the context of the foundations of quantum mechanics [1] and are an important resource for quantum measurement techniques [2]. However, cat states are notoriously vulnerable to perturbations caused by interactions with the environment. Even when the interactions are very weak, cat states can easily be destroyed due to their highly entangled nature. The state change can be measured by the fidelity between the original state and the resulting state, or by the purity of the resulting state. To recover the original cat state, we could employ a quantum error-correcting scheme [3], which, however, requires a macroscopic number of redundant qubits. 
In this paper, we propose a probabilistic scheme to recover a Schrödinger cat state, based on the concept of physical reversibility in quantum measurement [4, 5]. A quantum measurement is said to be physically reversible if there exists a reversing operation that recovers the premeasurement state from the postmeasurement state by means of a physical process with a nonzero probability of success. Since an interaction with the environment can be modeled as a kind of quantum measurement, we may expect that the reversing operation could recover the original cat state in a probabilistic way. However, the interaction with the environment differs from a quantum measurement in that it does not refer to the outcome of the "measurement", and this ignorance usually leads to decoherence of the system. Can the physical reversibility be employed to recover the original cat state even in this situation? The previous papers [4, 5, 6, 7, 8, 9, 10, 11] on physical reversibility have not addressed this question. We here give an affirmative answer. That is, our scheme provides a considerable increase in the fidelity together with an increase in the purity when the interaction of the system with the environment is weak. The reversing operation invokes a quantum measurement with postselection and thus has a probabilistic nature. However, the probability of success is high and the reversing operation increases both the mean fidelity and the mean purity. We shall describe an explicit model using spin systems with an Ising-type interaction, and discuss a possible experimental situation.

This paper is organized as follows. Section 2 develops a general theory of reversing operation. Section 3 considers an example of a Schrödinger cat state. Section 4 summarizes our results.

\section{General Theory of Reversing Operation}

Consider that a system with an initial pure density operator $\hat{\rho}_{\mathrm{s}}=|\psi\rangle\langle\psi|$, where the state $|\psi\rangle$ is assumed to be unknown to us, evolves in time according to the Hamiltonian $\hat{H}_{\mathrm{s}}$, and that the system is disturbed due to its interaction with the environment. Let the initial density operator of the environment, the Hamiltonian of the environment, and the interaction Hamiltonian be $\hat{\rho}_{\mathrm{e}}$, $\hat{H}_{\mathrm{e}}$, and $\hat{H}_{\mathrm{s} ; \mathrm{e}}$, respectively. Then, the state of the system after time $t_{\mathrm{e}}$ is described by a reduced density operator

$$
\hat{\rho}_{\mathrm{s}}^{\prime}=\operatorname{Tr}_{\mathrm{e}}\left[e^{-i \hat{H} t_{\mathrm{e}}}\left(\hat{\rho}_{\mathrm{s}} \otimes \hat{\rho}_{\mathrm{e}}\right) e^{i \hat{H} t_{\mathrm{e}}}\right]
$$


where $\hat{H} \equiv \hat{H}_{\mathrm{s}}+\hat{H}_{\mathrm{e}}+\hat{H}_{\mathrm{s} ; \mathrm{e}}$ denotes the total Hamiltonian and $\operatorname{Tr}_{\mathrm{e}}$ denotes the partial trace over the environment. According to the quantum operation formalism [3], there exists a set of linear operators $\left\{\hat{E}_{k}\right\}$ such that $\hat{\rho}_{\mathrm{s}}^{\prime}$ is related to $\hat{\rho}_{\mathrm{s}}$ by

$$
\hat{\rho}_{\mathrm{s}}^{\prime}=\sum_{k} \hat{E}_{k} \hat{\rho}_{\mathrm{s}} \hat{E}_{k}^{\dagger}
$$

where $\hat{E}_{k}$ 's satisfy

$$
\sum_{k} \hat{E}_{k}^{\dagger} \hat{E}_{k}=\hat{I}
$$

with $\hat{I}$ being the identity operator. Since $\hat{E}_{k}$ is a linear operator, it can uniquely be decomposed by the polar decomposition into

$$
\hat{E}_{k}=\hat{\mathcal{U}}_{k} \sqrt{\hat{E}_{k}^{\dagger} \hat{E}_{k}}
$$

where $\hat{\mathcal{U}}_{k}$ is a unitary operator. When the interaction with the environment is weak, $\sqrt{\hat{E}_{k}^{\dagger} \hat{E}_{k}}$ should be expanded as

$$
\sqrt{\hat{E}_{k}^{\dagger} \hat{E}_{k}} \simeq a_{k}\left(\hat{I}+g \hat{\epsilon}_{k}^{(1)}+g^{2} \hat{\epsilon}_{k}^{(2)}\right)
$$

where $a_{k}$ is a positive number, $g$ is a real dimensionless small parameter characterizing the strength of the interaction between the system and the environment, and $\hat{\epsilon}_{k}^{(1)}$ and $\hat{\epsilon}_{k}^{(2)}$ are Hermitian operators. On the other hand, the unitary operator $\hat{\mathcal{U}}_{k}$ can, in general, be written as

$$
\hat{\mathcal{U}}_{k} \simeq e^{i \gamma_{k}} e^{i g \hat{\Gamma}_{k}} \equiv e^{i \gamma_{k}} \hat{U}_{k}
$$

where $\gamma_{k}$ is a real number and $\hat{\Gamma}_{k}$ is a Hermitian operator. Note that $g \hat{\Gamma}_{k}$ is not necessarily small even if the interaction is weak due to the large degrees of freedom of the system and the environment. The weak interaction only implies that $\hat{U}_{k}$ does not depend strongly on $k$. Then, $\hat{U}_{k}$ can be decomposed into

$$
\hat{U}_{k}=e^{i g \hat{\Gamma}_{k}} \simeq e^{i g \hat{\Gamma}} e^{i g^{2} \hat{\delta}_{k}^{(2)}}
$$

where $g \hat{\Gamma}$ is a large Hermitian operator with no dependence on $k$, and $g^{2} \hat{\delta}_{k}^{(2)}$ is a small Hermitian operator. Thus for the case of a weak interaction, we obtain

$$
\hat{E}_{k} \simeq a_{k} e^{i \gamma_{k}} \hat{U}_{k}\left(\hat{I}+g \hat{\epsilon}_{k}^{(1)}+g^{2} \hat{\epsilon}_{k}^{(2)}\right)
$$


with

$$
\hat{U}_{k}^{\dagger} \hat{U}_{k^{\prime}} \simeq \hat{I}-i g^{2}\left(\hat{\delta}_{k}^{(2)}-\hat{\delta}_{k^{\prime}}^{(2)}\right)
$$

It follows from Eq. (3) that

$$
\begin{aligned}
& \sum_{k} a_{k}^{2}=1, \\
& \sum_{k} a_{k}^{2} \hat{\epsilon}_{k}^{(1)}=0, \\
& \sum_{k} a_{k}^{2}\left[\left(\hat{\epsilon}_{k}^{(1)}\right)^{2}+2 \hat{\epsilon}_{k}^{(2)}\right]=0 .
\end{aligned}
$$

The crucial observation here is that for the weak interaction, $\hat{E}_{k}$ has an approximate bounded left inverse and therefore fulfills the condition of physical reversibility [4, 5]. In fact, to an accuracy of order $g$, the left inverse of $\hat{E}_{k}$ can be written as

$$
\hat{E}_{k}^{-1} \simeq a_{k}^{-1} e^{-i \gamma_{k}}\left(\hat{I}-g \hat{\epsilon}_{k}^{(1)}\right) \hat{U}_{k}^{\dagger}
$$

It should be emphasized that such a weak interaction with the environment can profoundly disturb a Schrödinger cat state due to the effect of $\hat{U}_{k}$. The extent to which the state of the system is disturbed can be evaluated quantitatively in terms of the fidelity of $\hat{\rho}_{\mathrm{s}}^{\prime}$ to $\hat{\rho}_{\mathrm{s}}=|\psi\rangle\langle\psi|, F^{\prime} \equiv \sqrt{\left\langle\hat{\rho}_{\mathrm{s}}^{\prime}\right\rangle}$, where $\langle\hat{O}\rangle \equiv\langle\psi|\hat{O}| \psi\rangle$. It is estimated to be

$$
F^{\prime 2} \simeq \sum_{k} a_{k}^{2}\left|\left\langle\hat{U}_{k}\right\rangle\right|^{2}
$$

where the small terms $g \hat{\epsilon}_{k}^{(1)}$ and $g^{2} \hat{\epsilon}_{k}^{(2)}$ are ignored. Note that we cannot expand $\hat{U}_{k}$ in terms of $g$, since $g \hat{\Gamma}_{k}$ in Eq. (7) is, in general, not small. This means that the value of $F^{\prime}$ can be almost 0 for a cat state even if $g$ is small. The state of the system can thus be altered drastically by the interaction with the environment, however weak it is. On the other hand, the extent to which the state of the system becomes mixed can be quantified by the purity of the system, $P^{\prime} \equiv \operatorname{Tr} \hat{\rho}_{\mathrm{s}}^{\prime 2}$, which is estimated to be

$$
P^{\prime} \simeq 1-2 g^{2} \sum_{k} a_{k}^{2}\left\langle\left(\Delta \hat{\epsilon}_{k}^{(1)}\right)^{2}\right\rangle
$$


using Eqs. (8) $-(12)$, where $\Delta \hat{O} \equiv \hat{O}-\langle\hat{O}\rangle$. The purity thus does not decrease so drastically as the fidelity.

We now discuss the reversing operation to recover the original state $\hat{\rho}_{\mathrm{s}}$ from $\hat{\rho}_{\mathrm{s}}^{\prime}$. As a simplest example, we consider the "average" of the unitary operators $\left\{\hat{U}_{k}^{\dagger}\right\}$,

$$
\hat{\bar{U}}^{\dagger}=\sum_{k} a_{k}^{2} \hat{U}_{k}^{\dagger}
$$

since each outcome $k$ occurs with probability $p_{k} \simeq a_{k}^{2}$ by the "measurement" done by the environment. We note that this operator is approximately unitary for the case of weak interactions. In fact, given Eq. (9), we can easily show that

$$
\hat{\bar{U}}^{\dagger} \hat{\bar{U}} \simeq \hat{I}
$$

By applying the average unitary operator (16) to the system, $\hat{\rho}_{\mathrm{s}}^{\prime}$ is changed into

$$
\begin{aligned}
\hat{\rho}_{\mathrm{s}, \mathrm{u}}^{\prime \prime} & \equiv \hat{\bar{U}}^{\dagger} \hat{\rho}_{\mathrm{s}}^{\prime} \hat{\bar{U}} \\
& \simeq \hat{\rho}_{\mathrm{s}}+g^{2} \sum_{k} a_{k}^{2}\left(\hat{\epsilon}_{k}^{(2)} \hat{\rho}_{\mathrm{s}}+\hat{\rho}_{\mathrm{s}} \hat{\epsilon}_{k}^{(2)}+\hat{\epsilon}_{k}^{(1)} \hat{\rho}_{\mathrm{s}} \hat{\epsilon}_{k}^{(1)}\right)
\end{aligned}
$$

where Eqs. (9) -(11) are used. Thus, up to the first order in $g$, the original state is recovered by the reversing operation (16). Accordingly, the reversing operation can increase the fidelity to

$$
F_{\mathrm{u}}^{\prime \prime 2} \equiv\left\langle\hat{\rho}_{\mathrm{s}, \mathrm{u}}^{\prime \prime}\right\rangle \simeq 1-g^{2} \sum_{k} a_{k}^{2}\left\langle\left(\Delta \hat{\epsilon}_{k}^{(1)}\right)^{2}\right\rangle
$$

However, it cannot increase the purity,

$$
P_{\mathrm{u}}^{\prime \prime} \equiv \operatorname{Tr} \hat{\rho}_{\mathrm{s}, \mathrm{u}}^{\prime \prime 2}=P^{\prime}
$$

since a unitary operation cannot change the purity of a system. Therefore, to increase the purity as well as the fidelity, we must exploit the nonunitary aspect of the reversing operation. However, since we assume that we are ignorant about the state $|\psi\rangle$, we cannot use the projector $|\psi\rangle\langle\psi|$ to project the system to $|\psi\rangle$. Even if we could, it would generally be difficult to experimentally realize the projection onto the cat state due to its highly entangled nature. We will thus need a method for determining the nonunitary state reduction without the knowledge of $|\psi\rangle$. 
To implement this, consider a situation in which a probe with initial density operator $\hat{\rho}_{\mathrm{p}}$ and Hamiltonian $\hat{H}_{\mathrm{p}}$ interacts with the system during time $t_{\mathrm{p}}$ via an interaction Hamiltonian $\hat{H}_{\mathrm{s} ; \mathrm{p}}$, and then the probe is measured with respect to a complete set of projectors $\left\{\hat{P}_{m}\right\}$ associated with a certain observable. When the outcome of the probe measurement is $m$, the postmeasurement state of the system is given by

$$
\hat{\rho}_{\mathrm{s} \mid m}^{\prime \prime} \propto \operatorname{Tr}_{\mathrm{p}}\left[e^{-i \hat{H}^{\prime} t_{\mathrm{p}}}\left(\hat{\rho}_{\mathrm{s}}^{\prime} \otimes \hat{\rho}_{\mathrm{p}}\right) e^{i \hat{H}^{\prime} t_{\mathrm{p}}} \hat{P}_{m}\right]
$$

where $\hat{H}^{\prime}$ is the total Hamiltonian $\hat{H}^{\prime}=\hat{H}_{\mathrm{s}}+\hat{H}_{\mathrm{p}}+\hat{H}_{\mathrm{s} ; \mathrm{p}}$ and $\operatorname{Tr}_{\mathrm{p}}$ denotes the partial trace over the probe. According to a general formalism of quantum measurement [3], Eq. (21) can be rewritten with a set of linear operators $\left\{\hat{M}_{m}\right\}$ called measurement operators as

$$
\hat{\rho}_{\mathrm{s} \mid m}^{\prime \prime}=\frac{1}{p_{m}} \hat{M}_{m} \hat{\rho}_{\mathrm{s}}^{\prime} \hat{M}_{m}^{\dagger}
$$

with

$$
\sum_{m} \hat{M}_{m}^{\dagger} \hat{M}_{m}=\hat{I}
$$

where

$$
p_{m} \equiv \operatorname{Tr}\left[\hat{M}_{m} \hat{\rho}_{\mathrm{s}}^{\prime} \hat{M}_{m}^{\dagger}\right]
$$

is the probability for outcome $m$. Conversely, we can always construct a probe that accomplishes a quantum measurement described by a given set of operators $\left\{\hat{M}_{m}\right\}$ by appropriately choosing $\hat{\rho}_{\mathrm{p}}, \hat{H}_{\mathrm{p}}, \hat{H}_{\mathrm{s} ; \mathrm{p}}$, and $\left\{\hat{P}_{m}\right\}$. We here choose them so that for a particular outcome $m_{0}$, the corresponding measurement operator is given by

$$
\hat{M}_{m_{0}} \simeq b\left(\hat{I}+\zeta g^{2} \hat{\bar{\epsilon}}^{(2)}\right) \hat{\bar{U}}^{\dagger}
$$

where $b$ is a complex number, $\zeta$ is a positive number, and

$$
\hat{\bar{\epsilon}}^{(2)} \equiv \sum_{k} a_{k}^{2} \hat{\epsilon}_{k}^{(2)}
$$

is the average of $\left\{\hat{\epsilon}_{k}^{(2)}\right\}$. The other $\hat{M}_{m}$ 's are arbitrary as long as Eq. (23) is satisfied. The measurement operator (25) is the average unitary operator (16) followed by the average of the nonunitary part in Eq. (8), except for 
inclusion of a numerical factor of $\zeta$. Note that the average of $\left\{\hat{\epsilon}_{k}^{(1)}\right\}$ vanishes because of Eq. (11). The added nonunitary operator causes state reduction in almost the same way as the nonunitary operator in Eq. (8), purifying the state of the system. Substituting Eq. (25) into Eq. (24), we obtain

$$
p_{m_{0}} \simeq|b|^{2}\left[1+2 \zeta g^{2}\left\langle\hat{\bar{\epsilon}}^{(2)}\right\rangle\right] \text {. }
$$

The positive number $\zeta$ enhances the probability of obtaining the outcome $m_{0}$ and hence the effect of purification. Substituting Eqs. (25) and (27) into Eq. (22), we find

$$
\begin{aligned}
\hat{\rho}_{\mathrm{s} \mid m_{0}}^{\prime \prime} \simeq \hat{\rho}_{\mathrm{s}} & +g^{2} \sum_{k} a_{k}^{2}\left(\hat{\epsilon}_{k}^{(2)} \hat{\rho}_{\mathrm{s}}+\hat{\rho}_{\mathrm{s}} \hat{\epsilon}_{k}^{(2)}+\hat{\epsilon}_{k}^{(1)} \hat{\rho}_{\mathrm{s}} \hat{\epsilon}_{k}^{(1)}\right) \\
& +\zeta g^{2}\left(\Delta \hat{\bar{\epsilon}}^{(2)} \hat{\rho}_{\mathrm{s}}+\hat{\rho}_{\mathrm{s}} \Delta \hat{\bar{\epsilon}}^{(2)}\right) .
\end{aligned}
$$

Note that $\operatorname{Tr} \hat{\rho}_{\mathrm{s} \mid m_{0}}^{\prime \prime}=\operatorname{Tr} \hat{\rho}_{\mathrm{s}}=1$ holds because of Eq. (12). The reversing operation (25) also cancels the disturbance up to the order of $g$. Calculated up to the order of $g^{4}$ (see Appendix $\underline{\mathrm{A}}$ ), the fidelity $F_{m_{0}}^{\prime \prime} \equiv \sqrt{\left\langle\hat{\rho}_{\mathrm{s} \mid m_{0}}^{\prime \prime}\right\rangle}$ becomes

$$
\begin{gathered}
F_{m_{0}}^{\prime \prime 2}-F_{\mathrm{u}}^{\prime \prime 2} \simeq-\zeta^{2} g^{4}\left\langle\left(\Delta \hat{\bar{\epsilon}}^{(2)}\right)^{2}\right\rangle-\frac{1}{2} \zeta g^{4} \sum_{k} a_{k}^{2}\left[4\left\langle\Delta \hat{\epsilon}_{k}^{(1)} \Delta \hat{\bar{\epsilon}}^{(2)} \Delta \hat{\epsilon}_{k}^{(1)}\right\rangle\right. \\
\left.-\left\langle\Delta \hat{\bar{\epsilon}}^{(2)}\left(\Delta \hat{\epsilon}_{k}^{(1)}\right)^{2}\right\rangle-\left\langle\left(\Delta \hat{\epsilon}_{k}^{(1)}\right)^{2} \Delta \hat{\bar{\epsilon}}^{(2)}\right\rangle\right] .
\end{gathered}
$$

Up to the order of $g^{2}, F_{m_{0}}^{\prime \prime}$ is equal to $F_{\mathrm{u}}^{\prime \prime}$ given by Eq. (19). Thus, if we obtain the measurement outcome $m_{0}$, we can increase the fidelity as in the reversing operation (16). Moreover, we can increase the purity as well in the case of the reversing operation (25). The purity $P_{m_{0}}^{\prime \prime} \equiv \operatorname{Tr} \hat{\rho}_{\mathrm{s} \mid m_{0}}^{\prime \prime 2}$ satisfies

$$
\begin{aligned}
P_{m_{0}}^{\prime \prime}-P_{\mathrm{u}}^{\prime \prime}=P_{m_{0}}^{\prime \prime}-P^{\prime} & \simeq-4 \zeta g^{4} \sum_{k} a_{k}^{2}\left\langle\Delta \hat{\epsilon}_{k}^{(1)} \Delta \hat{\bar{\epsilon}}^{(2)} \Delta \hat{\epsilon}_{k}^{(1)}\right\rangle \\
& =2 \zeta g^{4} \sum_{k, k^{\prime}} a_{k}^{2} a_{k^{\prime}}^{2}\left\langle\Delta \hat{\epsilon}_{k}^{(1)} \Delta\left(\hat{\epsilon}_{k^{\prime}}^{(1)}\right)^{2} \Delta \hat{\epsilon}_{k}^{(1)}\right\rangle
\end{aligned}
$$

using Eq. (12). Although the increase in the purity is very small compared to 1 , its ratio to the lost purity by the environment is of the order of $g^{2}$, since

$$
R_{\mathrm{p}} \equiv \frac{P_{m_{0}}^{\prime \prime}-P^{\prime}}{1-P^{\prime}} \simeq \zeta \times O\left(g^{2}\right)
$$


from Eq. (15). It is this ratio that is relevant to an increase in decoherence time. That is, $R_{\mathrm{p}}$ increases the decoherence time from $\tau_{\mathrm{d}}$ to $\tau_{\mathrm{d}} /\left(1-R_{\mathrm{p}}\right)$. On the other hand, the fidelity is increased when $F^{\prime} \simeq 0$ to

$$
R_{\mathrm{f}} \equiv \frac{F_{m_{0}}^{\prime \prime}-F^{\prime}}{1-F^{\prime}} \simeq 1-O\left(g^{2}\right)
$$

which is independent of $\zeta$.

\section{System of Spin-1/2 Particles}

\subsection{Description of the model}

As a concrete example, we consider a system of $N_{s}(\equiv 2 s)$ spin- $1 / 2$ particles (or two-level systems), and assume that the system is in a cat state in which the spin states of the particles are either all up or all down along the $x$-axis,

$$
|\psi\rangle=c_{+}\left|\uparrow_{x}, \uparrow_{x}, \ldots, \uparrow_{x}\right\rangle+c_{-}\left|\downarrow_{x}, \downarrow_{x}, \ldots, \downarrow_{x}\right\rangle,
$$

where $\left|c_{+}\right|^{2}+\left|c_{-}\right|^{2}=1$. It is assumed that we have no a priori information about $c_{ \pm}$. With the spin operators of the $a$-th particle $\left\{\hat{s}_{x}^{(a)}, \hat{s}_{y}^{(a)}, \hat{s}_{z}^{(a)}\right\}$, the total spin operator of the system is given by $\hat{S}_{i}=\sum_{a=1}^{N_{s}} \hat{s}_{i}^{(a)}$, where $i=x, y, z$. Let $|s, \sigma\rangle$ be the simultaneous eigenstate of $\sum_{i} \hat{S}_{i}^{2}$ and $\hat{S}_{z}$ with eigenvalues $s(s+1)$ and $\sigma$, respectively. The density operator of the system is then written as

$$
\hat{\rho}_{\mathrm{s}}=|\psi\rangle\left\langle\psi\left|=\sum_{\sigma, \sigma^{\prime}} c_{\sigma} c_{\sigma^{\prime}}^{*}\right| s, \sigma\right\rangle\left\langle s, \sigma^{\prime}\right|,
$$

where

$$
c_{\sigma}=\frac{1}{2^{s}} \sqrt{\frac{(2 s) !}{(s+\sigma) !(s-\sigma) !}}\left[c_{+}+(-1)^{s-\sigma} c_{-}\right] .
$$

In the following discussions, we assume that $\hat{H}_{\mathrm{s}}=0$ for simplicity. The environment is assumed to consist of $N_{j}$ (三2j) spin-1/2 particles (or twolevel systems). The Hamiltonian of the environment is assumed to be

$$
\hat{H}_{\mathrm{e}}=-\Delta E \sum_{n=1}^{N_{j}} \hat{j}_{z}^{(n)}
$$


where $\Delta E$ is the energy difference between the spin states $|\uparrow\rangle_{n}$ and $|\downarrow\rangle_{n}$, and $\hat{j}_{z}^{(n)}$ is the spin $z$-component operator of the $n$-th particle of the environment. The spin of each particle is up with probability $\cos ^{2}(\theta / 2)$ and down with probability $\sin ^{2}(\theta / 2)$ along the $z$-axis, where $0 \leq \theta \leq \pi$. The environment is thus described by the density operator,

$$
\hat{\rho}_{\mathrm{e}}=\prod_{n=1}^{N_{j}}\left(\cos ^{2} \frac{\theta}{2}|\uparrow\rangle_{n n}\left\langle\uparrow\left|+\sin ^{2} \frac{\theta}{2}\right| \downarrow\right\rangle_{n n}\langle\downarrow|\right) .
$$

The interaction between the system and the environment is assumed to be

$$
\hat{H}_{\mathrm{s} ; \mathrm{e}}=\alpha_{\mathrm{e}} \sum_{n=1}^{N_{j}} \hat{j}_{z}^{(n)} \hat{S}_{z}
$$

where $\alpha_{\mathrm{e}}$ is a real constant.

The interaction with the environment acts as a random noise disturbance on the system when the state of the environment is traced over. After a certain period of time $t_{\mathrm{e}}$, we find that the density operator of the system is changed from $\hat{\rho}_{\mathrm{s}}$ to

$$
\hat{\rho}_{\mathrm{s}}^{\prime}=\sum_{\sigma, \sigma^{\prime}} c_{\sigma} c_{\sigma^{\prime}}^{*} N_{\sigma \sigma^{\prime}}^{(j)}(\theta)|s, \sigma\rangle\left\langle s, \sigma^{\prime}\right|
$$

where

$$
N_{\sigma \sigma^{\prime}}^{(j)}(\theta)=\left(e^{-i g\left(\sigma-\sigma^{\prime}\right)} \cos ^{2} \frac{\theta}{2}+e^{i g\left(\sigma-\sigma^{\prime}\right)} \sin ^{2} \frac{\theta}{2}\right)^{2 j}
$$

with an effective strength of interaction $g \equiv \alpha_{\mathrm{e}} t_{\mathrm{e}} / 2$. If the interaction is sufficiently weak, Eq. (40) can be approximated up to the order of $g^{2}$ as

$$
N_{\sigma \sigma^{\prime}}^{(j)}(\theta) \simeq e^{-g^{2} j \sin ^{2} \theta\left(\sigma-\sigma^{\prime}\right)^{2}-i 2 g j \cos \theta\left(\sigma-\sigma^{\prime}\right)} .
$$

However, because of the large $s$ and $j$, the weak interaction strength does not imply that the perturbation on the system is small. The fidelity of $\hat{\rho}_{\mathrm{s}}^{\prime}$ to $\hat{\rho}_{\mathrm{s}}$ is given by

$$
F^{\prime 2}=\sum_{\sigma, \sigma^{\prime}}\left|c_{\sigma}\right|^{2}\left|c_{\sigma^{\prime}}\right|^{2} \operatorname{Re}\left[N_{\sigma \sigma^{\prime}}^{(j)}(\theta)\right]
$$

Figure 1 shows $F^{\prime}$ as a function of $s$ for $c_{ \pm}=1 / \sqrt{2}, j=50, g=0.01$, and $\theta=\pi / 6$. The fidelity $F^{\prime}$ decreases as the degrees of freedom of the system 


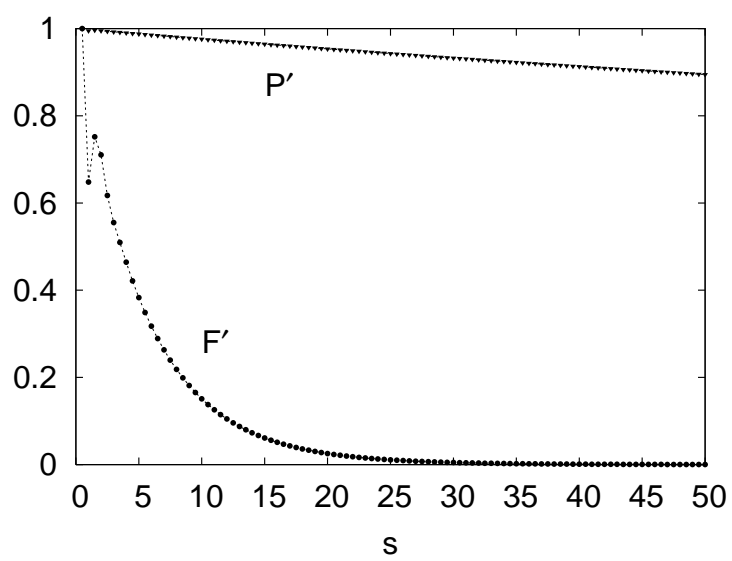

Figure 1: Fidelity $F^{\prime}$ and purity $P^{\prime}$ after an interaction with the environment as functions of spin $s$, which is equal to one half of the degrees of the system. The parameters used are $c_{ \pm}=1 / \sqrt{2}, j=50, g=0.01$, and $\theta=\pi / 6$. The fidelity $F^{\prime}$ oscillates near $s \simeq 1$ due to the effect of statistics (i.e., $s$ is an integer or a half-integer) which becomes negligible as $s$ becomes large.

$2 s$ become large. This decrease results from rapid oscillation of the cosine factor in

$$
\operatorname{Re}\left[N_{\sigma \sigma^{\prime}}^{(j)}(\theta)\right] \simeq e^{-g^{2} j \sin ^{2} \theta\left(\sigma-\sigma^{\prime}\right)^{2}} \cos \left[2 g j\left(\sigma-\sigma^{\prime}\right) \cos \theta\right],
$$

which represents the change in the relative phase between $|s, \sigma\rangle$ and $\left|s, \sigma^{\prime}\right\rangle$. The cosine factor oscillates with period $\pi / g j \cos \theta$ as a function of $\sigma-\sigma^{\prime}$, while the weight $\left|c_{\sigma}\right|^{2}\left|c_{\sigma^{\prime}}\right|^{2}$ concentrates near $\left(\sigma, \sigma^{\prime}\right)=(0,0)$ with width $\sqrt{s}$ owing to the binomial coefficient in Eq. (35). Since any oscillating function cancels out when it is averaged over the argument, we estimate that

$$
F^{\prime} \simeq 0
$$

if $s$ is so large that

$$
s \gtrsim \frac{\pi^{2}}{4 g^{2} j^{2} \cos ^{2} \theta} .
$$

Actually, as long as $s$ is large, the central limit theorem gives an analytic expression for the fidelity as (see Appendix B)

$$
F^{\prime 2} \sim \frac{1}{\sqrt{1+2 g^{2} s j \sin ^{2} \theta}} \exp \left[\frac{-2 g^{2} s j^{2} \cos ^{2} \theta}{1+2 g^{2} s j \sin ^{2} \theta}\right] .
$$


The interaction with the environment also causes the purity to degrade. The purity of $\hat{\rho}_{\mathrm{s}}^{\prime}$ is given by

$$
P^{\prime}=\sum_{\sigma, \sigma^{\prime}}\left|c_{\sigma}\right|^{2}\left|c_{\sigma^{\prime}}\right|^{2}\left|N_{\sigma \sigma^{\prime}}^{(j)}(\theta)\right|^{2} .
$$

Figure 1 shows $P^{\prime}$ as a function of $s$ for $c_{ \pm}=1 / \sqrt{2}, j=50, g=0.01$, and $\theta=\pi / 6$. The purity $P^{\prime}$ decreases as the degrees of freedom of the system $2 \mathrm{~s}$ become large, but this decrease is not so drastic as in the case of the fidelity, since $\left|N_{\sigma \sigma^{\prime}}^{(j)}(\theta)\right|^{2}$ involves no cosine factor; it is calculated to be

$$
\begin{aligned}
P^{\prime} & \simeq \sum_{\sigma, \sigma^{\prime}}\left|c_{\sigma}\right|^{2}\left|c_{\sigma^{\prime}}\right|^{2}\left[1-2 g^{2} j \sin ^{2} \theta\left(\sigma-\sigma^{\prime}\right)^{2}\right] \\
& =1-2 g^{2} s j \sin ^{2} \theta
\end{aligned}
$$

if $s$ is so small that

$$
s \ll \frac{1}{g^{2} j \sin ^{2} \theta} .
$$

Therefore, the cat state is drastically changed together with a slight degradation in the purity of the quantum state after the interaction with the environment. Moreover, we know neither $\hat{\rho}_{\mathrm{s}}$ nor $\hat{\rho}_{\mathrm{s}}^{\prime}$ due to our ignorance of $c_{ \pm}$. Nevertheless, these facts do not imply that the original cat state cannot be recovered.

As in Eq. (2), $\hat{\rho}_{\mathrm{s}}^{\prime}$ can be written in terms of $\left\{\hat{E}_{k}\right\}$ of the form of Eq. (8). We find that

$$
\begin{aligned}
a_{k} & =\frac{1}{2^{j}} \sqrt{\frac{(2 j) !}{(j+k) !(j-k) !}}, \\
\hat{U}_{k} & =e^{i g \hat{\Gamma}_{k}}=e^{-i 2 g j \cos \theta \hat{S}_{z}-i g^{2} k \sin 2 \theta \hat{S}_{z}^{2}}, \\
\hat{\epsilon}_{k}^{(1)} & =-2 k \sin \theta \hat{S}_{z}, \\
\hat{\epsilon}_{k}^{(2)} & =2\left(k^{2}-j\right) \sin ^{2} \theta \hat{S}_{z}^{2}, \\
\hat{\delta}_{k}^{(2)} & =-k \sin 2 \theta \hat{S}_{z}^{2}
\end{aligned}
$$

for $k=j, j-1, \ldots,-j+1,-j$. The condition (45) implies that $g \hat{\Gamma}_{k}$ is large for the cat state, while the condition (49) implies that $g \hat{\epsilon}_{k}^{(1)}$ and $g^{2} \hat{\epsilon}_{k}^{(2)}$ are 
small because of $\left\langle\hat{S}_{z}^{2}\right\rangle=s / 2$ and $\sum_{k} a_{k}^{2} k^{2}=j / 2$. The latter condition also implies that $\hat{U}_{k}$ does not strongly depend on $k$ as in Eq. (9), since

$$
\hat{U}_{k}^{\dagger} \hat{U}_{k^{\prime}} \simeq \hat{I}+i g^{2}\left(k-k^{\prime}\right) \sin 2 \theta \hat{S}_{z}^{2} .
$$

Below, we will concentrate on the case in which $s$ satisfies the following conditions:

$$
\frac{\pi^{2}}{4 g^{2} j^{2} \cos ^{2} \theta} \lesssim s \ll \frac{1}{g^{2} j \sin ^{2} \theta} .
$$

\subsection{Reversing operation}

To recover the cat state $\hat{\rho}_{\mathrm{s}}$, we perform a measurement [11] on the state $\hat{\rho}_{\mathrm{s}}^{\prime}$ using the information about the environment $(g, j, \theta)$. The probe of the measurement is a spin- $j$ system whose spin operators are $\left\{\hat{J}_{x}, \hat{J}_{y}, \hat{J}_{z}\right\}$ and Hamiltonian is $\hat{H}_{\mathrm{p}}=0$. The measurement proceeds as follows. The probe is first prepared in a coherent spin state $|\pi-\theta, \pi / 2\rangle$, i.e., the eigenstate of the spin component $\hat{J}_{y} \sin \theta-\hat{J}_{z} \cos \theta$ with eigenvalue $j$ [12]. The density operator of the probe is

$$
\hat{\rho}_{\mathrm{p}}=|\pi-\theta, \pi / 2\rangle\langle\pi-\theta, \pi / 2|,
$$

which represents a pure state in contrast with the state of the environment (37). The probe then interacts with the system via an interaction Hamiltonian

$$
\hat{H}_{\mathrm{s} ; \mathrm{p}}=\alpha_{\mathrm{p}} \hat{J}_{z} \hat{S}_{z}
$$

where $\alpha_{\mathrm{p}}$ is a real constant. The interaction is turned on during time $t_{\mathrm{p}}$ so that $\alpha_{\mathrm{p}} t_{\mathrm{p}} / 2=g$. After the interaction, a unitary operator

$$
\hat{U}_{\mathrm{p}}=e^{-i \pi \hat{J}_{y} / 2}
$$

is applied to the probe, and finally, the projective measurement on the probe observable $\hat{J}_{z}$ is performed. Let $m$ be the outcome of the measurement, where $m=j, j-1, \ldots,-j+1,-j$. The probability for obtaining outcome $m$ is

$$
p_{m}=\sum_{\sigma}\left|c_{\sigma}\right|^{2}\left|A_{m \sigma}^{(j)}(\theta)\right|^{2}
$$




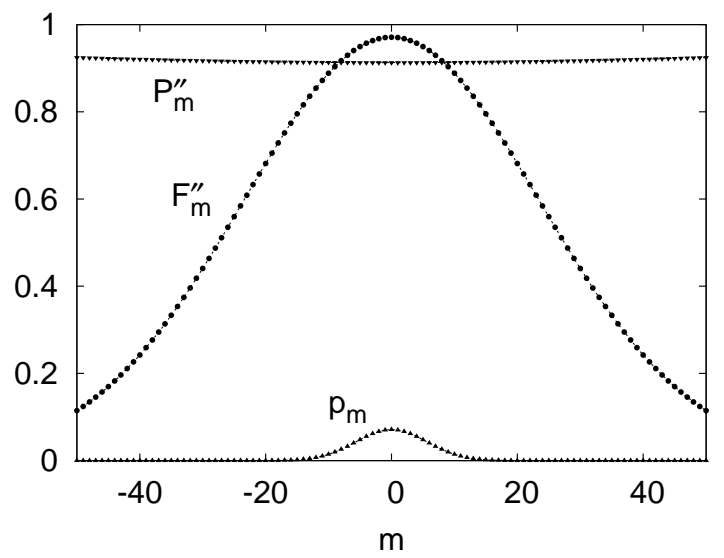

Figure 2: Probability $p_{m}$, fidelity $F_{m}^{\prime \prime}$, and purity $P_{m}^{\prime \prime}$ after a measurement as functions of outcome $m$ for $s=j=50, c_{ \pm}=1 / \sqrt{2}, g=0.01$, and $\theta=\pi / 6$. The mean fidelity and purity after the measurement are 0.945 and 0.913 , respectively.

where

$$
\begin{aligned}
& A_{m \sigma}^{(j)}(\theta) \equiv \frac{e^{-i j \pi / 2}}{2^{j}} \sqrt{\frac{(2 j) !}{(j+m) !(j-m) !}} \\
& \\
& \times\left(e^{-i g \sigma} \sin \frac{\theta}{2}+i e^{i g \sigma} \cos \frac{\theta}{2}\right)^{j-m} \\
& \times\left(e^{-i g \sigma} \sin \frac{\theta}{2}-i e^{i g \sigma} \cos \frac{\theta}{2}\right)^{j+m}
\end{aligned}
$$

The expectation value of outcome, $\sum_{m} m p_{m}$, is calculated to give 0 . Figure 2 shows $p_{m}$ as a function of $m$ for $s=j=50, c_{ \pm}=1 / \sqrt{2}, g=0.01$, and $\theta=\pi / 6$. The postmeasurement state is given by

$$
\hat{\rho}_{\mathrm{s} \mid m}^{\prime \prime}=\frac{1}{p_{m}} \sum_{\sigma, \sigma^{\prime}} c_{\sigma} c_{\sigma^{\prime}}^{*} N_{\sigma \sigma^{\prime}}^{(j)}(\theta) R_{m \sigma \sigma^{\prime}}^{(j)}(\theta)|s, \sigma\rangle\left\langle s, \sigma^{\prime}\right|,
$$

where $R_{m \sigma \sigma^{\prime}}^{(j)}(\theta)=A_{m \sigma}^{(j)}(\theta) A_{m \sigma^{\prime}}^{(j) *}(\theta)$.

As in Eq. (22), the measurement is described by measurement operators

$$
\hat{M}_{m}=\sum_{\sigma} A_{m \sigma}^{(j)}(\theta)|s, \sigma\rangle\langle s, \sigma|
$$


with $m=j, j-1, \ldots,-j+1,-j$. The particular outcome $m_{0}$ in Sec. 2 is the expectation value of outcome $m=0$. In fact, when expanded up to the second order of $g$, the operator $\hat{M}_{0}$ has the form of Eq. (25) with

$$
\begin{aligned}
& b=\frac{e^{-i j \pi / 2}}{2^{j}} \sqrt{\frac{(2 j) !}{j ! j !}}, \\
& \zeta=2,
\end{aligned}
$$

since

$$
\begin{gathered}
\hat{\bar{\epsilon}}^{(2)}=-j \sin ^{2} \theta \hat{S}_{z}^{2}, \\
\hat{\bar{U}}^{\dagger}=e^{i 2 g j \cos \theta \hat{S}_{z}},
\end{gathered}
$$

from the definitions (16) and (26) with Eqs. (150), (51), and (53).

The fidelity of $\hat{\rho}_{\mathrm{s} \mid m}^{\prime \prime}$ to $\hat{\rho}_{\mathrm{s}}$ is

$$
\begin{aligned}
F_{m}^{\prime \prime 2} & \equiv\left\langle\hat{\rho}_{\mathrm{s} \mid m}^{\prime \prime}\right\rangle \\
& =\frac{1}{p_{m}} \sum_{\sigma, \sigma^{\prime}}\left|c_{\sigma}\right|^{2}\left|c_{\sigma^{\prime}}\right|^{2} \operatorname{Re}\left[N_{\sigma \sigma^{\prime}}^{(j)}(\theta) R_{m \sigma \sigma^{\prime}}^{(j)}(\theta)\right],
\end{aligned}
$$

and, in particular, the fidelity at the expectation value $m=0$ is

$$
F_{0}^{\prime \prime 2} \simeq 1-g^{2} s j \sin ^{2} \theta
$$

using the expansions of $p_{m}, N_{\sigma \sigma^{\prime}}^{(j)}(\theta)$, and $R_{m \sigma \sigma^{\prime}}^{(j)}(\theta)$ up to the order of $g^{2}$. The mean squared fidelity and the mean fidelity are given by

$$
\begin{aligned}
\sum_{m} p_{m} F_{m}^{\prime \prime 2} & \simeq 1-2 g^{2} s j \sin ^{2} \theta \\
\sum_{m} p_{m} F_{m}^{\prime \prime} & \simeq 1-g^{2} s j \sin ^{2} \theta
\end{aligned}
$$

The fidelity $F_{m}^{\prime \prime}$ is also shown in Fig. 2 as a function of $m$ when $s=j=50$, $c_{ \pm}=1 / \sqrt{2}, g=0.01$, and $\theta=\pi / 6$. In this case, even though the effect of the environment is to decrease the fidelity to $F^{\prime}=2.41 \times 10^{-4}$, the reversing measurement recovers it to $F_{0}^{\prime \prime}=0.971$ when the most probable outcome $m=$ 0 is obtained by the measurement. This means that $97.1 \%$ of the lost fidelity is recovered by the measurement. Even if $m \neq 0$, the fidelity is still recovered 
as long as $m \simeq 0$, which occurs with a high probability. In fact, the mean fidelity after the measurement, $\sum_{m} p_{m} F_{m}^{\prime \prime}$, is 0.945 . Under the condition (56), the fidelity is drastically reduced by the interaction with the environment but is almost recovered by the reversing measurement. Surprisingly, such a drastic change occurs with a weak interaction. This is due to the large degrees of freedom being involved in the cat state.

On the other hand, the purity $P_{m}^{\prime \prime} \equiv \operatorname{Tr} \hat{\rho}_{\mathbf{s} \mid m}^{\prime \prime 2}$ is calculated to be

$$
\begin{aligned}
P_{m}^{\prime \prime} & =\frac{1}{p_{m}^{2}} \sum_{\sigma, \sigma^{\prime}}\left|c_{\sigma}\right|^{2}\left|c_{\sigma^{\prime}}\right|^{2}\left|N_{\sigma \sigma^{\prime}}^{(j)}(\theta) R_{m \sigma \sigma^{\prime}}^{(j)}(\theta)\right|^{2} \\
& \simeq 1-2 g^{2} s j \sin ^{2} \theta
\end{aligned}
$$

which is equal to $P^{\prime}$ in Eq. (48) up to the second order of $g$, independently of $m$. However, Eq. (30) shows that the purity is indeed increased by the measurement with outcome $m=0$,

$$
P_{0}^{\prime \prime}-P^{\prime} \simeq 8 g^{4} s^{2} j^{2} \sin ^{4} \theta>0 .
$$

Its ratio to the lost purity by the environment is given by

$$
R_{\mathrm{p}}=\frac{P_{0}^{\prime \prime}-P^{\prime}}{1-P^{\prime}} \simeq 4 g^{2} s j \sin ^{2} \theta .
$$

The purity $P_{m}^{\prime \prime}$ is shown in Fig. 2 as a function of $m$ for $s=j=50, c_{ \pm}=$ $1 / \sqrt{2}, g=0.01$, and $\theta=\pi / 6$. The effect of the environment lowers the purity to $P^{\prime}=0.895$, but the measurement with outcome $m=0$ recovers it to $P_{0}^{\prime \prime}=0.913$. Therefore, $R_{\mathrm{p}}$ is equal to 0.172 , indicating that $17.2 \%$ of the lost purity is recovered by the reversing measurement. The measurement can thus increase the decoherence time by about $20.7 \%$. The mean purity

$$
\sum_{m} p_{m} P_{m}^{\prime \prime} \simeq 1-2 g^{2} s j \sin ^{2} \theta
$$

is also increased to 0.913 by the measurement as in the case of fidelity.

For comparison, let us examine the degree to which we can restore the original state by performing the average unitary operation (67) on state $\hat{\rho}_{\mathrm{s}}^{\prime}$. The resulting state is given by

$$
\hat{\rho}_{\mathrm{s}, \mathrm{u}}^{\prime \prime}=\sum_{\sigma, \sigma^{\prime}} c_{\sigma} c_{\sigma^{\prime}}^{*} N_{\sigma \sigma^{\prime}}^{(j)}(\theta) e^{i 2 g j \cos \theta\left(\sigma-\sigma^{\prime}\right)}|s, \sigma\rangle\left\langle s, \sigma^{\prime}\right| .
$$


The unitary operation can recover the fidelity like the measurement. In fact, Eq. (29) shows that the fidelity $F_{\mathrm{u}}^{\prime \prime}$ is the same as $F_{0}^{\prime \prime}$ up to the order of $g^{5}$,

$$
F_{0}^{\prime \prime 2}-F_{\mathrm{u}}^{\prime \prime 2} \simeq O\left(g^{6}\right)
$$

For the previous example $\left(s=j=50, c_{ \pm}=1 / \sqrt{2}, g=0.01\right.$, and $\left.\theta=\pi / 6\right)$, the unitary operation also increases the fidelity to $F_{\mathrm{u}}^{\prime \prime}=0.971$. Unlike the measurement, however, the unitary operation cannot increase the purity. The purity $P_{\mathrm{u}}^{\prime \prime}$ is equal to $P^{\prime}$ and thus satisfies

$$
P_{0}^{\prime \prime}-P_{\mathrm{u}}^{\prime \prime} \simeq 8 g^{4} s^{2} j^{2} \sin ^{4} \theta>0
$$

as in Eq. (75).

\subsection{Feasibility of the reversing operation}

To perform the reversing operation, we must know the parameters of the environment, such as the effective strength of the interaction $g$, the number of particles $2 j$, and the probability for spin-up state $\cos ^{2}(\theta / 2)$. From the energy difference $\Delta E$ in Eq. (36), we can estimate $\theta$ when the environment is in thermal equilibrium at temperature $T$ by using the relation

$$
\tan ^{2} \frac{\theta}{2}=e^{-\Delta E / k_{\mathrm{B}} T}
$$

where $k_{\mathrm{B}}$ is the Boltzmann constant. On the other hand, to estimate $g$ and $j$, we regard $F^{\prime}$ and $P^{\prime}$ as functions of time $t_{\mathrm{e}}$, since they depend on $t_{\mathrm{e}}$ through $g=\alpha_{\mathrm{e}} t_{\mathrm{e}} / 2$. Let $t_{0}$ be the time when $F^{\prime}$ becomes 0. From Eqs. (45) and (48), we obtain

$$
\begin{aligned}
g_{0}^{2} j^{2} & \simeq \frac{\pi^{2}}{4 s \cos ^{2} \theta}, \\
g_{0}^{2} j & \simeq \frac{1-P_{0}^{\prime}}{2 s \sin ^{2} \theta},
\end{aligned}
$$

where $g_{0}$ and $P_{0}^{\prime}$ are $g$ and $P^{\prime}$ at $t_{0}$, respectively. Combining these relations, we can estimate $j, g_{0}$, and $g=g_{0} t_{\mathrm{e}} / t_{0}$.

Finally, we describe a possible experimental situation for the reversing operation [11]. Consider the system as an ensemble of $2 s$ two-level atoms and the probe as an ensemble of $2 j$ photons with two polarizations (horizontal 
or vertical). The photons can then be regarded as a spin- $j$ system using the spin operators

$$
\begin{aligned}
& \hat{J}_{x} \equiv \frac{1}{2}\left(\hat{a}_{1}^{\dagger} \hat{a}_{2}+\hat{a}_{2}^{\dagger} \hat{a}_{1}\right), \\
& \hat{J}_{y} \equiv \frac{1}{2 i}\left(\hat{a}_{1}^{\dagger} \hat{a}_{2}-\hat{a}_{2}^{\dagger} \hat{a}_{1}\right), \\
& \hat{J}_{z} \equiv \frac{1}{2}\left(\hat{a}_{1}^{\dagger} \hat{a}_{1}-\hat{a}_{2}^{\dagger} \hat{a}_{2}\right),
\end{aligned}
$$

where $\hat{a}_{1}$ and $\hat{a}_{2}$ are the annihilation operator for photons with horizontal and vertical polarizations, respectively. The initial probe state (57) is prepared by subjecting horizontally polarized photons to a half-wave plate and a phase shifter, and the interaction (58) is realized by using the paramagnetic Faraday rotation [13, 14. The unitary operator (59) corresponds to a half-wave plate and the projective measurement of $\hat{J}_{z}$ is achieved by two photodetectors for the two polarizations. Typically, in this situation, $s \sim 10^{8}, j \sim 10^{8}$, and $g \sim 10^{-8}$. Therefore, the condition (56) is satisfied if $\theta^{2} \ll 1$, which means $k_{\mathrm{B}} T \ll \Delta E / \ln 4$.

\section{Conclusion}

We have proposed a probabilistic reversing operation that can recover both the fidelity and purity after they are deteriorated through weak interactions with the environment. Since there is no unitary operation that can increase the purity, the reversing operation must involve a nonunitary state reduction of a quantum measurement. We have considered an ensemble of spin- $1 / 2$ particles in a Schrödinger cat state as a system and another ensemble of spin$1 / 2$ particles in a mixed state as an environment. The cat state of the system is then destroyed by a weak Ising-type interaction with the environment. The fidelity to the original cat state is drastically decreased due to the large degrees of freedom of the system and environment, despite a slight decrease in the purity of the system. We have also shown that a reversing operation can achieve a profound recovery of the fidelity together with a nonzero increase in the purity with a high probability of success. The reversing operation is achieved by a quantum measurement that uses a probe. The probe is a spin system in a coherent spin state and interacts with the system via an Ising-type Hamiltonian. If the measurement ends with a preferred outcome, 
we can increase not only the fidelity but also the purity by the reversing operation.

We have also discussed a physical implementation of our model using twolevel atoms as a system and photons as a probe. Since the interaction would be feasible in view of recent advances in experimental techniques, the reversing operation could be experimentally realized in the near future. Although in this paper we have focused on the reversing operation of a Schrödinger cat state undergoing an Ising-type interaction, our scheme could equally be applied to other general states undergoing a large class of weak interactions.

\section{Acknowledgments}

This research was supported by a Grant-in-Aid for Scientific Research (Grant No. 17071005) from the Ministry of Education, Culture, Sports, Science and Technology of Japan.

\section{Appendix}

\section{A Fourth-Order Calculation}

We here outline the derivation of Eqs. (29) and (30). To calculate the fidelity and the purity to the order of $g^{4}$, one might think that Eqs. (8), (9), and (25) should be expanded to the order of $g^{4}$ as

$$
\begin{aligned}
\hat{E}_{k} & \simeq a_{k} e^{i \gamma_{k}} \hat{U}_{k}\left(\hat{I}+g \hat{\epsilon}_{k}^{(1)}+g^{2} \hat{\epsilon}_{k}^{(2)}+g^{3} \hat{\epsilon}_{k}^{(3)}+g^{4} \hat{\epsilon}_{k}^{(4)}\right), \\
\hat{U}_{k}^{\dagger} \hat{U}_{k^{\prime}} & \simeq \hat{I}-i g^{2}\left(\hat{\delta}_{k}^{(2)}-\hat{\delta}_{k^{\prime}}^{(2)}\right)+g^{3} \hat{\delta}_{k k^{\prime}}^{(3)}+g^{4} \hat{\delta}_{k k^{\prime}}^{(4)}, \\
\hat{M}_{m_{0}} & \simeq b\left(\hat{I}+\zeta g^{2} \hat{\bar{\epsilon}}^{(2)}+g^{3} \hat{\chi}^{(3)}+g^{4} \hat{\chi}^{(4)}\right) \hat{\bar{U}}^{\dagger}
\end{aligned}
$$

where $\hat{\epsilon}_{k}^{(3)}, \hat{\epsilon}_{k}^{(4)}, \hat{\delta}_{k k^{\prime}}^{(3)}, \hat{\delta}_{k k^{\prime}}^{(4)}, \hat{\chi}^{(3)}$, and $\hat{\chi}^{(4)}$ are Hermitian operators. However, we can show that such higher-order terms are irrelevant to the calculation of $F_{m_{0}}^{\prime \prime 2}-F_{\mathrm{u}}^{\prime \prime 2}$ and $P_{m_{0}}^{\prime \prime}-P_{\mathrm{u}}^{\prime \prime}$ to the order of $g^{4}$. The proof goes as follows. We first consider the contribution of $\hat{\chi}^{(4)}$. In doing so, we can ignore the other operators, since $\hat{\chi}^{(4)}$ is of the fourth order itself. We then obtain

$$
\hat{\rho}_{\mathrm{s} \mid m_{0}}^{\prime \prime} \simeq \hat{\rho}_{\mathrm{s}}+g^{4}\left(\Delta \hat{\chi}^{(4)} \hat{\rho}_{\mathrm{s}}+\hat{\rho}_{\mathrm{s}} \Delta \hat{\chi}^{(4)}\right),
$$


which results in $F_{m_{0}}^{\prime \prime}=P_{m_{0}}^{\prime \prime}=1$. Therefore, $\hat{\chi}^{(4)}$ does not contribute to the fidelity and the purity to the order of $g^{4}$. Similarly, $\hat{\chi}^{(3)}$ does not do so to the order of $g^{3}$. Although $\hat{\chi}^{(3)}$ may appear in the fourth order together with $\hat{\epsilon}_{k}^{(1)}$, such a term vanishes because of Eq. (111) by the summation over $k$. From these findings, we can set

$$
\hat{\chi}^{(3)}=\hat{\chi}^{(4)}=0 .
$$

On the other hand, if $\hat{\bar{\epsilon}}^{(2)}=0, F_{m_{0}}^{\prime \prime 2}-F_{\mathrm{u}}^{\prime \prime 2}=P_{m_{0}}^{\prime \prime}-P_{\mathrm{u}}^{\prime \prime}=0$, since $\hat{M}_{m_{0}}$ then reduces to the average unitary operation (16). This means that each term of $F_{m_{0}}^{\prime \prime 2}-F_{\mathrm{u}}^{\prime \prime 2}$ and $P_{m_{0}}^{\prime \prime}-P_{\mathrm{u}}^{\prime \prime}$ must contain at least one $\hat{\bar{\epsilon}}^{(2)}$. Since $\hat{\bar{\epsilon}}^{(2)}$ is of the second order, we can set

$$
\hat{\delta}_{k k^{\prime}}^{(3)}=\hat{\delta}_{k k^{\prime}}^{(4)}=\hat{\epsilon}_{k}^{(3)}=\hat{\epsilon}_{k}^{(4)}=0 .
$$

Consequently, in order to calculate $F_{m_{0}}^{\prime \prime 2}-F_{\mathrm{u}}^{\prime \prime 2}$ and $P_{m_{0}}^{\prime \prime}-P_{\mathrm{u}}^{\prime \prime}$ to the order of $g^{4}$, it is sufficient to use Eqs. (8), (9), and (25). Then, we can straightforwardly derive Eqs. (29) and (30).

\section{B Degradation of Fidelity}

We here explain the derivation of Eq. (46). From Eq. (35), the weight $\left|c_{\sigma}\right|^{2}$ in Eq. (42) is given by

$$
\left|c_{\sigma}\right|^{2}=\frac{1}{2^{2 s}} \frac{(2 s) !}{(s+\sigma) !(s-\sigma) !}\left[1+(-1)^{s-\sigma}\left(c_{+} c_{-}^{*}+c_{+}^{*} c_{-}\right)\right] .
$$

When $s$ is large, the term with factor $(-1)^{s-\sigma}$ can be ignored, since it is canceled by the summation over $\sigma$ due to

$$
\sum_{\sigma} \frac{1}{2^{2 s}} \frac{(2 s) !}{(s+\sigma) !(s-\sigma) !}(-1)^{s-\sigma} \sigma^{n}=0
$$

for $n=0,1, \ldots, 2 s-1$. The weight $\left|c_{\sigma}\right|^{2}$ is then a binomial distribution whose mean and variance are 0 and $s / 2$, respectively. The central limit theorem (or equivalently Stirling's formula) states that as $s$ increases, the binomial distribution becomes close to a normal distribution with the mean and variance unaltered:

$$
\left|c_{\sigma}\right|^{2} \simeq \frac{1}{2^{2 s}} \frac{(2 s) !}{(s+\sigma) !(s-\sigma) !} \rightarrow \frac{1}{\sqrt{\pi s}} \exp \left(-\frac{\sigma^{2}}{s}\right) .
$$


At the same time, the summation over $\sigma$ is replaced with the integral over $\sigma$

$$
\sum_{\sigma=-s}^{s} \rightarrow \int_{-\infty}^{\infty} d \sigma
$$

since $\sigma$ is now considered as a continuous variable from $-\infty$ to $\infty$. Using Eq. (41), the fidelity (42) can be written as

$$
\begin{aligned}
F^{\prime 2} \sim & \frac{1}{\pi s} \iint d \sigma d \sigma^{\prime} \exp \left[-\frac{1}{s}\left(\sigma^{2}+\sigma^{\prime 2}\right)\right] \\
& \times \exp \left[-g^{2} j \sin ^{2} \theta\left(\sigma-\sigma^{\prime}\right)^{2}-i 2 g j \cos \theta\left(\sigma-\sigma^{\prime}\right)\right],
\end{aligned}
$$

which gives Eq. (46) through the Gaussian integral.

\section{References}

[1] A. J. Leggett, Prog. Theor. Phys. Suppl. 69, 80 (1980).

[2] V. Giovannetti, S. Lloyd, and L. Maccone, Science 306, 1330 (2004).

[3] M. A. Nielsen and I. L. Chuang, Quantum Computation and Quantum Information (Cambridge University Press, Cambridge, 2000).

[4] M. Ueda, N. Imoto, and H. Nagaoka, Phys. Rev. A 53, 3808 (1996).

[5] M. Ueda, in Frontiers in Quantum Physics: Proceedings of the International Conference on Frontiers in Quantum Physics, Kuala Lumpur, Malaysia, 199\%, edited by S. C. Lim, R. Abd-Shukor, and K. H. Kwek (Springer-Verlag, Singapore, 1999), pp. 136-144.

[6] M. Ueda and M. Kitagawa, Phys. Rev. Lett. 68, 3424 (1992).

[7] A. Imamoḡlu, Phys. Rev. A 47, R4577 (1993).

[8] A. Royer, Phys. Rev. Lett. 73, 913 (1994); 74, 1040(E) (1995).

[9] M. Koashi and M. Ueda, Phys. Rev. Lett. 82, 2598 (1999).

[10] H. Terashima and M. Ueda, Int. J. Quantum Inf. 3, 633 (2005).

[11] H. Terashima and M. Ueda, Phys. Rev. A 74, 012102 (2006). 
[12] F. T. Arecchi, E. Courtens, R. Gilmore, and H. Thomas, Phys. Rev. A 6, 2211 (1972).

[13] A. Kuzmich, L. Mandel, J. Janis, Y. E. Young, R. Ejnisman, and N. P. Bigelow, Phys. Rev. A 60, 2346 (1999).

[14] Y. Takahashi, K. Honda, N. Tanaka, K. Toyoda, K. Ishikawa, and T. Yabuzaki, Phys. Rev. A 60, 4974 (1999). 